\title{
Facile synthesis of multiporous CuCo2O4 microspheresas efficient electrocatalysts for rechargeable Li-O2 batteries
}

\author{
Pengfa Li ${ }^{\mathrm{a}}$, Wang Sun ${ }^{\mathrm{a}^{*}}$, Jinshuo Qiao ${ }^{\mathrm{a}}$, Zhenhua Wang ${ }^{\mathrm{a}}$ and Kening Sun ${ }^{\mathrm{a}, \mathrm{b}^{*}}$ \\ ${ }^{a}$ Beijing Key Laboratory for Chemical Power Source and Green Catalysis, School of Chemical \\ Engineering and Environmental, Beijing Institute of Technology, Beijing, 100081, People's Republic \\ of China; \\ ${ }^{b}$ Collaborative Innovation Center of Electric Vehicles in Beijing, No.5 Zhongguancun South Avenue, \\ Haidian District, Beijing, 100081, People's Republic of China \\ abitkeningsun@163.com, bsunwang@bit.edu.cn.
}

KEYWORD: Lithium-oxygen battery; Multiporous; Electrocatalyst; Structural.

\begin{abstract}
Multiporous CuCo2O4 microspheres have been synthesized via a facile solvothermal method and used as bifunctional electrocatalysts for rechargeable Li-O2 batteries. The Li-O2 battery utilizing CuCo2O4 shows a higher specific capacity of $5607 \mathrm{mAh}$ g- 1 than that with pure Ketjen black (KB). Moreover, the Cu-Co2O4-based electrode enables much enhanced cyclability than that of the carbon-only cathode. Such excellent catalytic performance of CuCo2O4 could be associated with its inherent catalytic activity and multi-porous microspheres structure.
\end{abstract}

\section{INTRODUCTION}

Rechargeable lithium-oxygen batteries have received a great deal of interest in recent years due to its extremely high specific energy, which make them promising energy storage devices for electric vehicles [Abraham K.M.,1996][Girishkumar G.,2010]. A typical nonaqueous rechargeable Li-oxygen battery is composed of $\mathrm{Li}$ metal as the negative electrode, a $\mathrm{Li}$ ion conducting nonaqueous electrolyte, and porous oxygen diffusion cathode. The basic chemical reactions of non-aqueous electrolyte $\mathrm{Li}-\mathrm{O} 2$ batteries involve the formation of $\mathrm{Li} 2 \mathrm{O} 2$ during the discharge process (oxygen reduction reaction, ORR) and the decomposition of $\mathrm{Li} 2 \mathrm{O} 2$ during the charge process (oxygen evolution reaction, OER) [Peng Zhangquan,2012]. However, there are a great barrier to making Li air battery practical, including low round-trip efficiency, low rate capability, and poor cycling stability [ Girishkumar G.,2010].

Recently, great efforts have been made to improve the charge performance of Li-oxygen batteries by employing bifunctional catalyst in oxygen electrode. These catalysts mainly include noble metals [Peng Zhangquan,2012][ Lei Yu,2013], metal nitrides [Shui Jiang-Lan,2012], and also various classes of metal oxides [Xu Ji-Jing,2013][ Lee Jin-Hyon,2012].

Among those catalysts, covalent hybrids of spinel metal oxides have higher electrochemical activities as catalysts for Li-O2 batteries. Cobalt oxides have drawn the most potential because of reasonably good catalytic activity, low cost, ease of preparation and good chemical stability. However, due to the toxicity and high cost of cobalt, one of the worthwhile efforts is to partially replace the $\mathrm{Co}$ in $\mathrm{Co} 3 \mathrm{O} 4$ by cheaper and more eco-friendly alternative metals.

Spinel CuCo2O4 nanocrystals have been investigated as a cathode material in Li-oxygen batteries [Liu Ying,2014]. The authors found that the CuCo2O4/KB electrode exhibits much lower polarization, better rate capability and longer cycling life. In the simulated air conditions, the battery delivers a high capacity of $7962 \mathrm{mAh}$ g- 1 with a discharge-recharge voltage gap of $0.95 \mathrm{~V}$ at $50 \mathrm{~mA} \mathrm{~g}-1$.

Multiporous materials, which not only provide more electrocatalytic sites but also promote mass transport (oxygen and ions) in the electrolyte have been reported as electrocatalysts for rechargeable Li-O2 batteries [Ma Shunchao,2013].

In this paper, multiporous $\mathrm{CuCo} 2 \mathrm{O} 4$ microspheres were synthesized via a facile solvothermal method and used as the electrocatalyst for Li-O2 batteries. Its multiporous structure maximizes the 
availability of the catalytic sites and facilitates the diffusion of electrons and reactants. The Li-O2 batteries which use $\mathrm{CuCo} 2 \mathrm{O} 4$ microspheres as electrocatalyst display higher discharge capacity and excellent cycling performance.

\section{EXPERIMENTAL}

\subsection{Synthesis of materials}

All the chemical reagents were analytical grade and used without further purification. CuCo2O4 microspheres were prepared through a modified one-step solvothermal method. In a typical procedure [Ma Shunchao,2013], $1 \mathrm{mmol} \mathrm{CuCl} 2 \cdot 2 \mathrm{H} 2 \mathrm{O}$ and $2 \mathrm{mmol} \mathrm{CoCl} 2 \cdot 6 \mathrm{H} 2 \mathrm{O}$ were dissolved into $30 \mathrm{~mL}$ of ethylene glycol (EG) and $10 \mathrm{~mL}$ of distilled water. After magnetic stirring for an hour, $30 \mathrm{mmol}$ of NH4HCO3 was added to the above mixture at room temperature. The resultant solution was continually stirred for an hour and transferred into a $50 \mathrm{~mL}$ Teflon-lined stainless steel autoclave and heated at $200{ }^{\circ} \mathrm{C}$ for $24 \mathrm{~h}$. The autoclave cooled to room temperature naturally. The precipitates were collected by centrifugation and washed with distilled water and ethanol three times, followed by vacuum-drying at $70{ }^{\circ} \mathrm{C}$ for $10 \mathrm{~h}$. The porous $\mathrm{CuCo} 2 \mathrm{O} 4$ microspheres were obtained by calcining at $400{ }^{\circ} \mathrm{C}$ for $4 \mathrm{~h}$ in air.

\subsection{Characterization of samples}

The samples were characterized by X-ray diffraction (Rigaku Ultima IV, $\mathrm{Cu} \mathrm{K} \alpha$ radiation, $40 \mathrm{kV}$, $40 \mathrm{~mA}$ ), scanning electron microscopy (SEM, QUANTA FEG 250) and transmission electron microscopy (JEOL JEM-2100F, Tokyo, Japan).

\subsection{Electrochemical measurements}

The Li-O2 batteries were measured using 2025-type coin battery. The oxygen cathodes were prepared by coating a homogenous ink composed of a mixture of $30 \mathrm{wt} \%$ CuCo2O4 catalyst, $60 \mathrm{wt} \%$ Ketjen black EC600JD (KB), $10 \mathrm{wt} \%$ polyvinylidene fluoride (PVDF) or $90 \mathrm{wt} \% \mathrm{~KB}, 10 \mathrm{wt} \%$ PVDF onto a current collector. The mass loading of the CuCo2O4/KB catalyst was $1.1 \mathrm{mg} \mathrm{cm}-2$. The cell was then assembled in an Ar-filled glovebox. The electrolyte was $1 \mathrm{M} \mathrm{LiCF3SO3} \mathrm{in}$ tetraethylene glycol dimethyl ether (TEGDME). The discharge/charge tests were controlled by a LAND-CT2001A tester under an oxygen atmosphere. Cyclic voltammetry (CV) was performed between 2.0 and $4.5 \mathrm{~V}$ using a CHI660 electrochemical station.

\section{RESULTS AND DISCUSSION}

The X-ray diffraction (XRD) patterns of CuCo2O4 are shown in Figure 1. The results are consistent with the spinel crystalline structure CuCo2O4 (JCPDS No. 23-1390). The distinct peaks on wide-angle XRD pattern are observed at $2 \theta$ values of $18.88,31.08,36.62,38.31,44.52,55.29$, 58.96 and 64.79 which correspond to (111), (220), (311), (222), (400), (422), (511), and (440) plane reflections of the spinel CuCo2O4 structure, respectively; no other diffraction peaks were found, which indicate that the products were composed of pure CuCo2O4. 


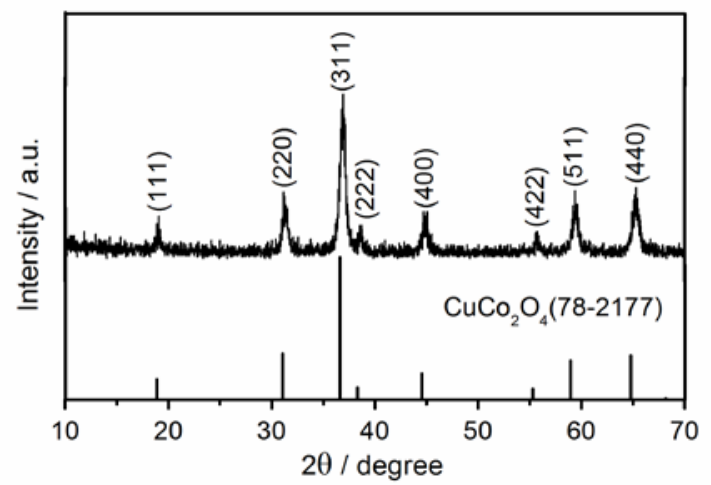

Figure 1. XRD patterns the $\mathrm{CuCo}_{2} \mathrm{O}_{4}$ microspheres.

As shown in Figure 2a, the product is composed of microspheres with diameter of 0.5-0.7 $\mu \mathrm{m}$. The microspheres are formed by the aggregation of nanoparticles with typical sizes ranging from 15 to $20 \mathrm{~nm}$. Furthermore, the TEM images (Figure 2b and 2c) obviously reveal that the microstructure of a CuCo2O4 sphere composed of numerous nanoparticles, and there are many mesopores that exist in the CuCo2O4 microstructure. The representative high resolution TEM (HRTEM) in Figure 2d displays distinct lattice fringes with d-spacing of $0.47 \mathrm{~nm}$ and $0.25 \mathrm{~nm}$, corresponding to the (111) and (311) planes of CuCo2O4, indicating that the samples were highly crystallized.

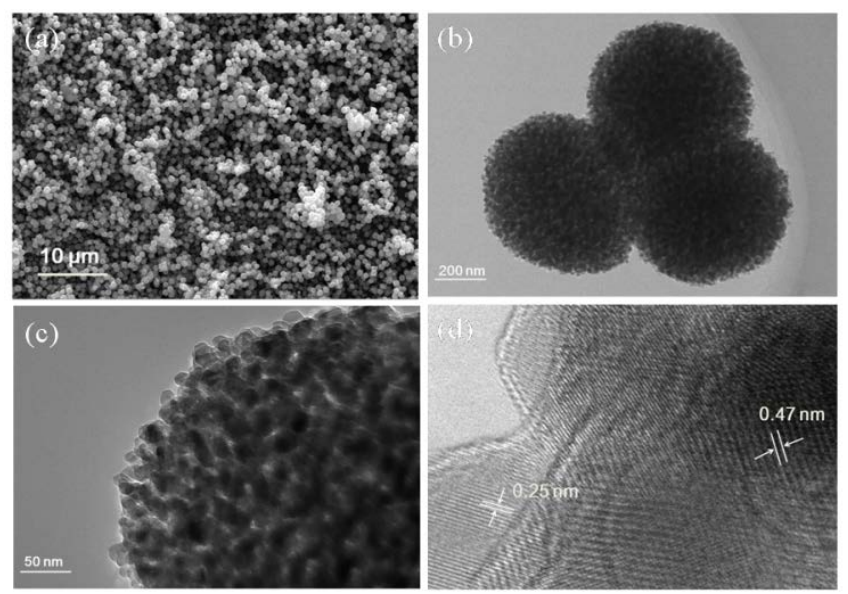

Figure 2. (a) SEM, (b, c) TEM and (d) HRTEM images of as-prepared CuCo2O4 microspheres.

Cyclic voltammetry (CV) was employed to investigate the ORR and OER activity of the synthesized CuCo2O4 microspheres in nonaqueous electrolyte as shown in Figure 3. The cyclic voltammogram shows a reduction peak, indicating the start of reaction at $2.92 \mathrm{~V}$ in the $\mathrm{CuCo} 2 \mathrm{O} 4 / \mathrm{KB}$ battery, while at $2.76 \mathrm{~V}$ in the KB battery. The $0.16 \mathrm{~V}$ higher shift in the reduction potential is attributed to the catalytic effect of $\mathrm{CuCo} 2 \mathrm{O} 4$ microspheres. Besides, the CuCo2O4/KB battery shows a much obvious current density during anodic scans $(>3.0 \mathrm{~V})$. These results demonstrate efficient catalytic activity of $\mathrm{CuCo} 2 \mathrm{O} 4$ for both the ORR and OER in aprotic media. 


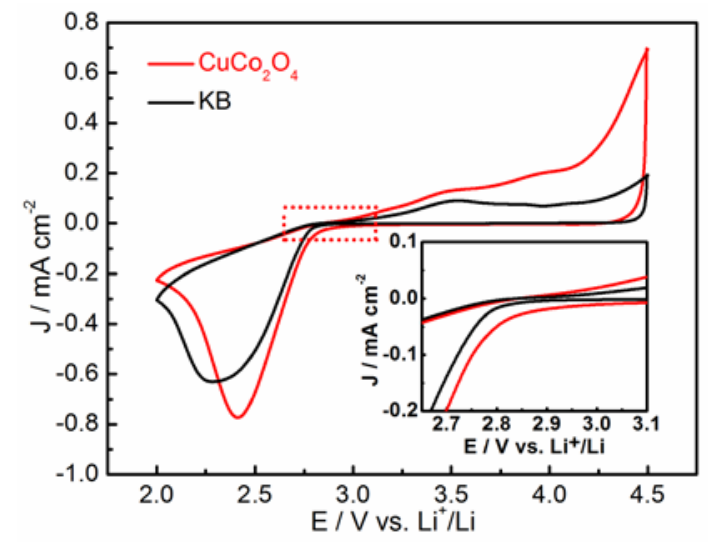

Figure 3. CV curves of CuCo2O4/KB and KB carbon-only electrode at a scan rate of $0.1 \mathrm{mV} \mathrm{s}-1$.

Given their favourable structures as demonstrated above, the multiporous CuCo2O4 microspheres were investigated as effective electrocatalysts for rechargeable Li-O2 batteries. Both the applied current density (mA g-1) and the achieved capacity (mAh g-1) were calculated based on the mass of carbon (KB). For the galvanostatic discharge/charge test, the lower and upper cut-off voltage is 2.2 and $4.4 \mathrm{~V}$ (vs. Li), respectively.

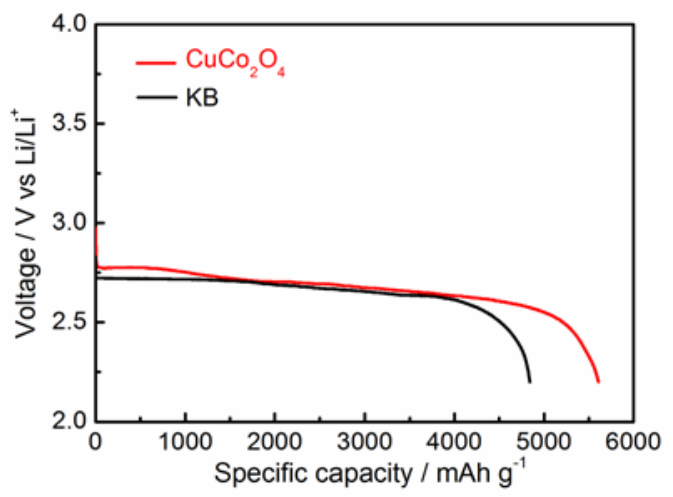

Figure 4. Discharge curves of Li-O2 batteries at a current density of $100 \mathrm{~mA}$ g-1 for CuCo2O4-based electrodes (a) and KB-based electrodes (b).

As can be seen in Figure 4, the Li-O2 battery with $\mathrm{CuCo} 2 \mathrm{O} 4$ electrode exerts specific capacities of $5607 \mathrm{mAh}$ g-1, which is higher than the battery with bare KB at a current density of $100 \mathrm{~mA}$ g-1. To avoid the decomposition of electrolyte on the discharge products during the charge/discharge processes, the capacity-limited cycling method that has been adopted in the literature was used in this work [Peng Zhangquan,2012]. The cycling performance of the CuCo2O4 cathode was tested at a current density of $100 \mathrm{~mA}$ g-1 under specific capacity limit of $500 \mathrm{mAh}$ g-1 (Figure 5a and 5b). Under this protocol, an energy density of $1350 \mathrm{Wh}$ kg-1 (500 mAh g-1 × 2.7 V) can be achieved, which is about two times the energy density of commercial cathodes in Li-ion batteries (640 Wh kg-1, 160 mAh g-1×4 V) [Lim Hee-Dae,2013]. The CuCo2O4 electrode could be discharged and charged stably for at least 23 cycles above $2.0 \mathrm{~V}$, on the contrary, the KB electrode can only keep less than 7 cycles. The improved cycling performance of $\mathrm{CuCo} 2 \mathrm{O} 4$ cathode can be ascribed to the inherent catalytic activity of spinel-type CuCo2O4 and its multiporous structure [Liu Ying,2014]. This multiporous structures ensuring can provide many electrocatalytic sites and also promote the flow of gases and infiltration of the electrolyte, and eventually improve the cyclability greatly [Wang Hailiang,2012]. 


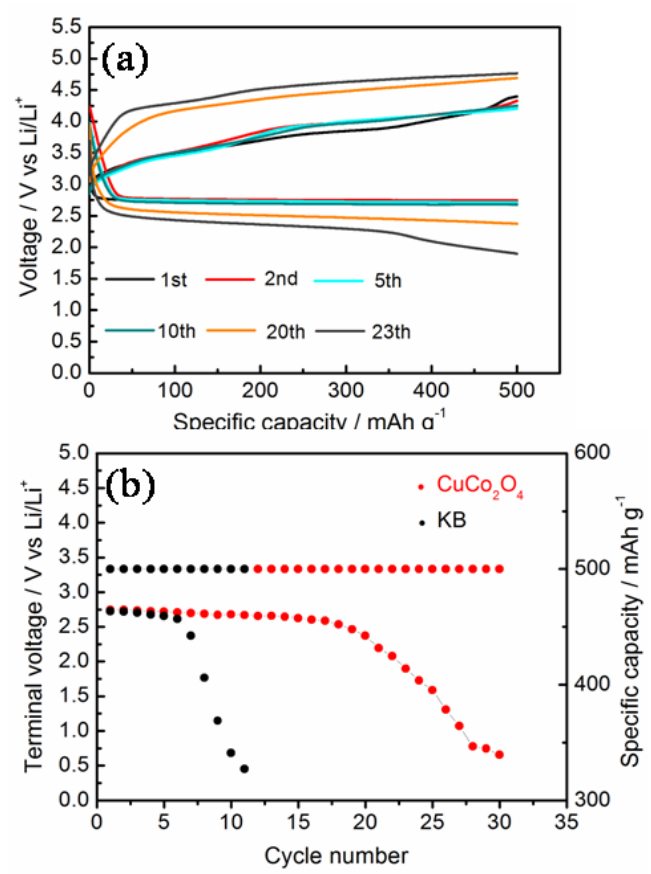

Figure 5. (a) Cycling response and (b) voltage of the terminal discharge vs cycle number of the $\mathrm{Li}-\mathrm{O} 2$ cell with the CuCo2O4/KB electrode under specific capacity limit of $500 \mathrm{mAh}$ g-1 at a current density of $100 \mathrm{~mA}$ g-1.

\section{CONCLUSIONS}

In summary, we successfully fabricated multiporous CuCo2O4 microspheres via a facile solvothermal method. These samples were investigated as electrocatalyst materials for lithium-oxygen battery applications. Compared with the carbon-only electrode, the CuCo2O4/C electrode exhibits a significantly enhanced discharge capacity and cycling performance. These results highlight the promising application of such a low-cost multiporous oxide for high performance Li-O2 batteries.

\section{ACKNOWLEDGEMENTS}

This work is financial supported by National Natural Science Foundation of China (Grant no. 21376001), Excellent Young Scholars Research Fund of Beijing Institute of Technology (Contract no. 2013YR1013) and Foundation for Basic Research of Beijing Institute of Technology (Contract no. 20141042003).

\section{REFERENCE}

[1] Abraham K.M. et al. 1996. A polymer electrolyte-based rechargeable lithium/oxygen battery. Journal of The Electrochemical Society 143(1):1-5.

[2] Girishkumar G. et al. 2010. Lithium-air battery: promise and challenges. Journal of Physical Chemistry Letters 1(14):2193-2203.

[3] Peng Zhangquan et al. 2012. A reversible and higher-rate $\mathrm{Li}_{2} \mathrm{O}_{2}$ Battery. Science 337(6094):563-566.

[4] Lei Yu et al. 2013. Synthesis of porous carbon supported palladium nanoparticle catalysts by atomic layer deposition: application for rechargeable lithium- $\mathrm{O}_{2}$ battery. Nano Letters 13(9): 4182-4189.

[5] Shui Jiang-Lan et al. 2012. Fe/N/C composite in $\mathrm{Li}^{-\mathrm{O}_{2}}$ battery: studies of catalytic structure and activity toward oxygen evolution reaction. Journal of the American Chemical Society 134(40): 16654-16661. 
[6] Xu Ji-Jing et al. 2013. Synthesis of perovskite-based porous $\mathrm{La}_{0.75} \mathrm{Sr}_{0.25} \mathrm{MnO}_{3}$ nanotubes as a highly efficient electrocatalyst for rechargeable lithium oxygen batteries. Angewandte Chemie-International Edition 52(14) 3887-3890.

[7] Lee Jin-Hyon et al. 2012. The role of vacancies and defects in $\mathrm{Na}_{0.44} \mathrm{MnO}_{2}$ nanowire catalysts for lithium-oxygen batteries. Energy \& Environmental Science 5(11)9558-9565.

[8] Ma Shunchao et al. 2013. Multiporous $\mathrm{MnCo}_{2} \mathrm{O}_{4}$ Microspheres as an Efficient Bifunctional Catalyst for Nonaqueous $\mathrm{Li}_{2} \mathrm{O}_{2}$ Batteries. Journal of Physical Chemistry $C$ 117(49):25890-25897.

[9] Lim Hee-Dae et al. 2013. A new catalyst-embedded hierarchical air electrode for high-performance $\mathrm{Li}_{-} \mathrm{O}_{2}$ batteries. Energy \& Environmental Science 6(12)3570-3575.

[10] Liu Ying et al. 2014. Facile synthesis of spinel $\mathrm{CuCo}_{2} \mathrm{O}_{4}$ nanocrystals as high-performance cathode catalysts for rechargeable Li-air batteries. Chemical Communications 50(93):14635-14638.

[11]Wang Hailiang et al. 2012. Rechargeable $\mathrm{Li}_{2} \mathrm{O}_{2}$ batteries with a covalently coupled $\mathrm{MnCo}_{2} \mathrm{O}_{4}$-graphene hybrid as an oxygen cathode catalyst. Energy \& Environmental Science 5(7) 7931-7935. 